\title{
Hot bottom burning and s-process nucleosynthesis in massive AGB stars at the beginning of the thermally-pulsing phase ${ }^{\star}$
}

\author{
D. A. García-Hernández ${ }^{1,2}$, O. Zamora ${ }^{1,2}$, A. Yagüe ${ }^{3,1,2}$, S. Uttenthaler ${ }^{4}$, A. I. Karakas ${ }^{5}$, M. Lugaro ${ }^{6}$, \\ P. Ventura ${ }^{3}$, and D. L. Lambert ${ }^{7}$ \\ 1 Instituto de Astrofísica de Canarias, C/ Via Láctea s/n, 38200 La Laguna, Spain \\ e-mail: agarcia@iac.es \\ 2 Departamento de Astrofísica, Universidad de La Laguna (ULL), 38206 La Laguna, Spain \\ 3 INAF-Osservatorio Astronomico di Roma, via Frascati 33, 00040 Monteporzio, Italy \\ ${ }^{4}$ University of Vienna, Department of Astrophysics, Türkenschanzstraße 17, 1180 Vienna, Austria \\ 5 Research School of Astronomy \& Astrophysics, Australian National University, ACT 2611 Canberra, Australia \\ ${ }^{6}$ Monash Centre for Astrophysics (MoCA), Monash University, 3800 Victoria, Australia \\ 7 W. J. McDonald Observatory. The University of Texas at Austin. 1 University Station, C1400. Austin, TX 78712-0259, USA \\ Received 2 May 2013 / Accepted 9 June 2013 \\ ABSTRACT

\begin{abstract}
We report the first spectroscopic identification of massive Galactic asymptotic giant branch (AGB) stars at the beginning of the thermal pulse (TP) phase. These stars are the most Li-rich massive AGBs found to date, super Li-rich AGBs with $\log \varepsilon(\mathrm{Li}) \sim 3-4$. The high Li overabundances are accompanied by weak or no s-process element (i.e. $\mathrm{Rb}$ and $\mathrm{Zr}$ ) enhancements. A comparison of our observations with the most recent hot bottom burning (HBB) and s-process nucleosynthesis models confirms that $\mathrm{HBB}$ is strongly activated during the first TPs but the ${ }^{22} \mathrm{Ne}$ neutron source needs many more TP and third dredge-up episodes to produce enough Rb at the stellar surface. We also show that the short-lived element Tc, usually used as an indicator of AGB genuineness, is not detected in massive AGBs, which is in agreement with the theoretical predictions when the ${ }^{22} \mathrm{Ne}$ neutron source dominates the s-process nucleosynthesis.
\end{abstract}

Key words. stars: AGB and post-AGB - stars: abundances - stars: evolution - nuclear reactions, nucleosynthesis, abundances stars: atmospheres - stars: late-type

\section{Introduction}

Low- and intermediate-mass $\left(0.8<M<8 M_{\odot}\right)$ stars end their lives with a phase of strong mass loss and thermal pulses (TP) on the asymptotic giant branch (AGB, Herwig 2005), and are one of the main contributors to the chemical enrichment (e.g. $\mathrm{C}, \mathrm{N}, \mathrm{Li}, \mathrm{F}$, and s-process elements) of the interstellar medium. After each TP the surface convective zone moves inwards, and may reach internal layers contaminated by $3 \alpha$ nucleosynthesis, enriched in $\mathrm{C}$. This mixing episode is known as third dredge-up (TDU). After many TDUs the surface $\mathrm{C}$ exceeds the $\mathrm{O}$ content, transforming originally $\mathrm{O}$-rich stars into $\mathrm{C}$-rich stars. However, this applies only to AGB stars in the mass range from $\sim 1.5$ to 3-4 $M_{\odot}$. More massive stars experience hot bottom burning (HBB, Sackmann \& Boothroyd 1992), i.e. proton-capture nucleosynthesis at the base of the outer envelope that favours the conversion of $\mathrm{C}$ to $\mathrm{N}$ by the $\mathrm{CN}$-cycle and reconversion of the $\mathrm{C}$-rich to an O-rich atmosphere. The HBB process is activated when the temperature at the bottom of the envelope reaches $40 \mathrm{MK}$, the same required to ignite the Cameron \& Fowler (1971) mechanism (Mazzitelli et al. 1999), by which great amounts of Li can be produced in the surface regions. The detection of Li overabundances in massive O-rich AGB stars both in our Galaxy (GarcíaHernández et al. 2007) and in the Magellanic Clouds (MCs; e.g. Plez et al. 1993; García-Hernández et al. 2009) has confirmed the activation of HBB in the more massive AGB stars.

Theoretical nucleosynthesis models also predict the presence of the elements (e.g. $\mathrm{Rb}, \mathrm{Zr}$, and $\mathrm{Sr}$ ) that can be made by slow neutron captures (the s process) and are dredged up to the stellar surface. According to the most recent models, ${ }^{13} \mathrm{C}(\alpha, n){ }^{16} \mathrm{O}$ is

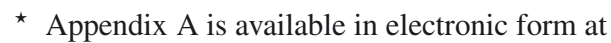
http://www . aanda. org the preferred neutron source for $\sim 1-3 M_{\odot}$, while for more massive stars neutrons are mainly released by ${ }^{22} \mathrm{Ne}(\alpha, n)^{25} \mathrm{Mg}$ (e.g. Karakas 2010; van Raai et al. 2012; Karakas et al. 2012). The ${ }^{22} \mathrm{Ne}$ neutron source requires higher temperatures and produces higher neutron densities than the ${ }^{13} \mathrm{C}$ source. The relative abundance of $\mathrm{Rb}$ to other nearby s-elements such as $\mathrm{Zr}$ is very sensitive to the neutron density owing to the operation of branchings in the s-process path at ${ }^{85} \mathrm{Kr}$ and ${ }^{86} \mathrm{Rb}$ (Lambert et al. 1995; Abia et al. 2001; van Raai et al. 2012). In this context, the [Rb/Zr] ratio is a powerful discriminant of the operation of the ${ }^{13} \mathrm{C}$ versus the ${ }^{22} \mathrm{Ne}$ neutron source in AGB stars.

A small group of Galactic stars showing $\mathrm{OH}$ maser emission, known as $\mathrm{OH} / \mathrm{IR}$ stars, have been identified as massive (4-8 $\left.M_{\odot}\right)$ O-rich HBB AGB stars showing strong Rb overabundances coupled with weak $\mathrm{Zr}$ enhancements (García-Hernández et al. 2006, 2007). This provides the first observational evidence that ${ }^{22} \mathrm{Ne}$ is indeed the dominant neutron source in massive Rb-rich AGB stars. However, this study was intentionally biased towards the redder and more extreme Galactic $\mathrm{OH} / \mathrm{IR}$ stars, which experience very strong mass-loss rates (up to $\sim 10^{-4}-10^{-5} M_{\odot} / \mathrm{yr}$ ) and are expected to be at the end of the thermally-pulsing (TP) AGB phase. A very advanced evolutionary AGB stage is also suggested by the large variations in the measured $\mathrm{Li}$ abundances $(-1.0 \leq \log \varepsilon(\mathrm{Li}) \leq 2.6$; see van Raai et al. 2012); the Lirich stars in that sample display $\mathrm{Li}$ abundances larger than solar, but significantly smaller than those found in a few super Li-rich Galactic AGB stars (with $\log \varepsilon(\mathrm{Li})>3-4$; e.g. Abia et al. 1999) ${ }^{1}$.

1 We note that a few super Li-rich AGBs were found in the MCs (e.g. Plez et al. 1993) but these are less massive $\left(\sim 3-5 M_{\odot}\right)$ Rb-poor HBB-AGBs where the ${ }^{13} \mathrm{C}$ neutron source dominates the s-process nucleosynthesis (see García-Hernández et al. 2006, 2009). 
Interestingly, HBB models predict that massive AGB stars experience a super Li-rich phase $(\log \varepsilon(\mathrm{Li}) \sim 4)$ at the beginning of the TP phase (e.g. Mazzitelli et al. 1999; van Raai et al. 2012). Yet, to date, no super Li-rich massive Galactic AGB stars have been unambiguously identified (see e.g. Uttenthaler \& Lebzelter 2010; Uttenthaler et al. 2011). In this Letter we report the first detections of super Li-rich massive AGB stars in our Galaxy. The extreme $\mathrm{Li}$ overabundances found together with the lack of s-process element enhancements are consistent with these stars being truly massive O-rich AGB stars at the beginning of the TP phase.

\section{Sample, optical observations, and chemical abundance analysis}

Our sample is composed of the Galactic disc O-rich AGB stars RU Cyg, SV Cas, R Cen, and RU Ari. Their AGB status is deduced from their relatively long pulsation periods ( 200-500 days), large amplitude variability, late spectral type (>M5), and infrared excess (see García-Hernández et al. 2007). All stars show similar infrared excesses and IRAS colours (R Cen and RU Ari are the bluest and reddest sources, respectively), but very different circumstellar maser emission from O-based molecules such as $\mathrm{SiO}, \mathrm{H}_{2} \mathrm{O}$, and $\mathrm{OH}$. In Table A.1 we list the four AGB stars together with their Galactic coordinates, present-day variability periods ${ }^{2}$, and maser information. An evolutionary sequence of increasing mass-loss from $\mathrm{SiO}$, to $\mathrm{H}_{2} \mathrm{O}$ and $\mathrm{OH}$ maser emission is expected in these stars (Lewis 1989; Habing 1996). Thus, the OH/IR star RU Ari is presumably more evolved (and/or more massive) than the other three AGBs for which no $\mathrm{OH}$ maser emission has been detected. Also, defining marks of the three non-OH/IR AGBs are their complex (e.g. double maxima) and changing variability properties. The dominant period of the Mira-like star R Cen has decreased from 500 days in 2000 (Hawkins et al. 2001) to the present value of 251 days while the other two stars have increased their dominant periods from $\sim 200-300$ days to $\sim 450$ days. It has been speculated that the changing period is related to a recent TP in AGB stars (see e.g. Wood \& Zarro 1981; Uttenthaler et al. 2011).

The optical observations of the stars RU Cyg, SV Cas, and RU Ari presented here are part of our recent high-resolution $(R \sim$ $60000)$ optical spectroscopic survey of a large sample $(\sim 100)$ of relatively blue Galactic O-rich AGB stars not previously studied. This survey has been carried out in several observing runs from 2006 to 2008 by using the Tull (Tull et al. 1995) and SARG spectrographs at the $2.7 \mathrm{~m}$ Harlan J. Smith (HJS) Telescope and at the Telescopio Nazionale Galileo, respectively; the optical spectra and the abundance analysis for the whole sample will be presented in a future paper. In particular, the optical spectra reported here were taken in the wavelength range $\sim 3650-9500 \AA(R \sim$ 60000 with the $1.2^{\prime \prime}$ slit and the grating E2) at the HJS telescope. On the other hand, the unpublished high-resolution $(R \sim 50000)$ optical spectrum of R Cen was taken with the ESO-VLT UVES spectrograph (with the $0.7^{\prime \prime}$ slit), covering the $~ 3770-4900$, 6670-8470, and 8650-10500 ̊ spectral regions. The star R Cen was observed within the ESO programme 65.L-0317(A) that was devoted to studying AGB stars in the Galactic bulge (GB; see e.g. Uttenthaler et al. 2007). However, R Cen is a lowlatitude $\left(b=+1.2^{\circ}\right)$ Galactic disc AGB star and displays a Li I $6708 \AA$ line that is much stronger than in the AGBs studied

\footnotetext{
2 Present-day periods have been determined by us from AAVSO (http://www.aavso.org) visual light curves (Uttenthaler et al. 2011).
}

by Uttenthaler et al. (2007). The exposure times varied between a few seconds (R Cen) and 3-5 min (RU Cyg and SV Cas) for the bluer AGBs and 20 minutes for the more obscured star RU Ari. A signal-to-noise ratio (S/N) of $\geq 30-50$ at $4200 \AA$ and in excess of 100 at wavelengths longer than $5900 \AA$ was achieved in the blue AGBs. The only exception was the redder AGB star RU Ari for which a S/N higher than 10 was achieved at $5924 \AA$ (the Tc I line) and higher than 40 at $\lambda>6400 \AA$.

The two-dimensional optical echelle spectra were reduced to single-order one-dimensional spectra using standard astronomical tasks. We are mainly interested in the wavelength regions around the Li I $6708 \AA$ and $\mathrm{Rb}$ I $7800 \AA$ resonance lines and around the $\mathrm{ZrO} 6474 \AA$ bandhead. The Galactic O-rich AGB stars analysed here are bright enough in the 4000-6000 region, permitting us to search for the Tc absorption lines (e.g. at $\sim 4238,4262,4297$, and $5924 \AA$ ) for the first time. This is in strong contrast with the more obscured $(\mathrm{OH} / \mathrm{IR})$ massive $\mathrm{Rb}$-rich AGB stars previously studied in our Galaxy (García-Hernández et al. 2006, 2007) that are too faint at wavelengths below $6000 \AA$. Indeed, the blue AGBs in our sample do not display the still unidentified molecular bands that are clearly present in obscured massive Rb-rich AGB spectra (e.g. at 7400-7600 A; see García-Hernández et al. 2009). These unidentified molecular bands are also detected in the much redder $(\mathrm{OH} / \mathrm{IR}) \mathrm{AGB}$ star RU Ari. This spectroscopic difference permit us to doublecheck the $\mathrm{Zr}$ abundance derived from the $\mathrm{ZrO}$ molecular bands with that estimated from the lack of the $\mathrm{Zr}$ I lines in the 7400-7600 A region in the blue AGBs (see below).

The chemical abundance analysis follows the procedure previouly used by us in Galactic O-rich AGB stars (see GarcíaHernández et al. 2006, 2007 for more details). In short, we used the latest version (v12.1.1) of the TURBOSPECTRUM package (Alvarez \& Plez 1998; Plez 2012) with line-blanketed model atmospheres and up-to-date molecular/atomic linelists to construct a grid of synthetic spectra appropiate for cool O-rich AGB stars in the Galactic disc: $T_{\text {eff }}=2600-3500 \mathrm{~K}$ in steps of $100 \mathrm{~K}$, $F W H M=200-600 \mathrm{~m} \AA$ in steps of $50 \mathrm{~m} \AA, \log g=-0.5$, $\xi=3 \mathrm{~km} \mathrm{~s}^{-1}$, and solar metallicity $([\mathrm{M} / \mathrm{H}]=0.0)$.

The observations were compared to the synthetic spectra in the regions 6455-6499, 6670-6730, and 7775-7835 $\AA$, covering the ZrO $6474 \AA$ A bandhead, the Li I $6708 \AA$ line, and the Rb I $7800 \AA$ line (Figs. A.1 and A.2). The best fit to the TiO bandheads and the pseudocontinuum around the atomic lines provided the $T_{\text {eff }}$, which was found to be $3000 \pm 100 \mathrm{~K}$ for all sample stars. The abundances (or upper limits) of $\mathrm{Li}, \mathrm{Rb}$, and $\mathrm{Zr}$ were obtained by adjusting the $\mathrm{Li} \mathrm{I}$ and $\mathrm{Rb}$ I lines and the $\mathrm{ZrO}$ molecular bands, respectively. In the blue AGBs, we note that the $\mathrm{Zr}$ abundance derived from the $\mathrm{ZrO}$ molecular bands is consistent with the lack of the $\mathrm{Zr}$ I lines in the 7400-7600 А region. The spectroscopic $T_{\text {eff }}$ and abundances are given in Table A.1, where we also list the abundance uncertainties estimated for each star. The three bluer AGB stars show strong Li enhancements $(\log \varepsilon(\mathrm{Li}) \geq 2$ ) together with no (or small) $\mathrm{Rb}$ and $\mathrm{Zr}$ overabundances $(\log \varepsilon(\mathrm{Rb}, \mathrm{Zr}) \sim 2.6 \pm 0.3)$. However, the redder AGB RU Ari displays a very high Rb content accompanied by no $\mathrm{Li}$ and no (or weak) $\mathrm{Zr}$ (see Sect. 4).

Finally, we have searched for the presence of the strong Tc I lines at $\sim 4238,4262$, and $4297 \AA$ and the much weaker Tc I $5924 \AA$ line by following the method described by Uttenthaler et al. (2011). We note that the bluest AGB R Cen was not observed around the Tc I $5924 \AA$ line and the reddest AGB star RU Ari is too faint around the stronger Tc I lines 
D. A. García-Hernández et al.: Early massive AGBs
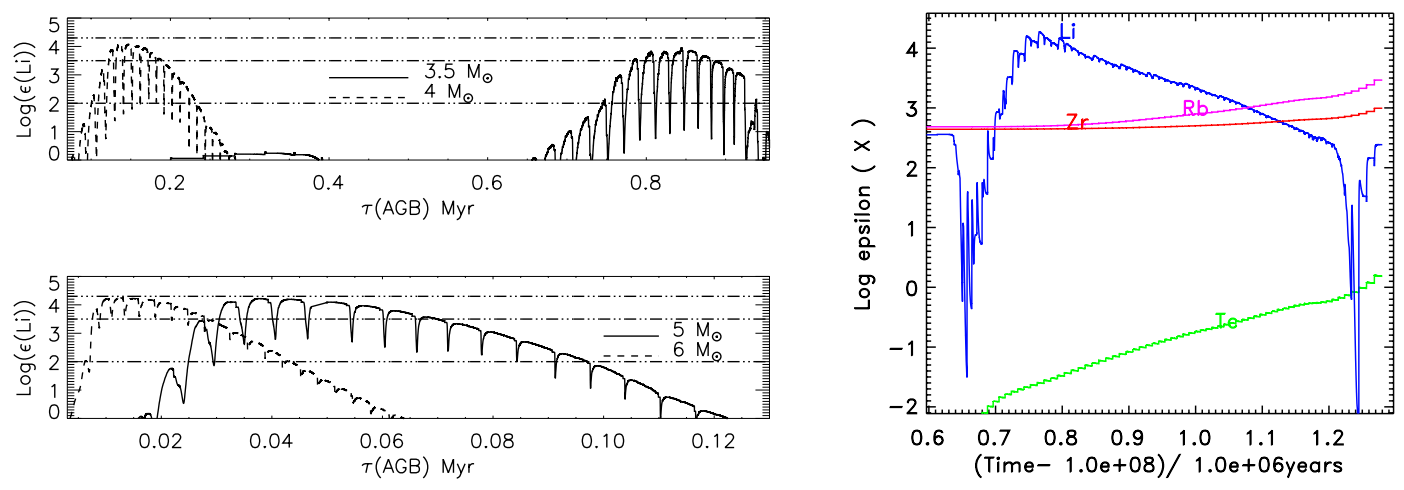

Fig. 1. Left panel: HBB-FST predictions of the Li abundance versus time from the beginning of the AGB (in Myr). The horizontal lines represent the observed values of $\log \varepsilon(\mathrm{Li})=2.0,3.5$, and 4.3. Right panel: $\mathrm{HBB}-\mathrm{MLT} \mathrm{Li}, \mathrm{Rb}, \mathrm{Zr}$, and Tc predictions for a $5 M_{\odot}$ model with a delayed superwind. We note that the Tc abundance is an upper limit because the trend of the half-life of ${ }^{99} \mathrm{Tc}-$ decreasing with temperature from terrestrial $0.22 \mathrm{Myr}$ to $\sim 3 \mathrm{yr}$ at the peak temperature of $\sim 350 \mathrm{MK}-$ is not included in the model (though we do not expect large differences, see Mathews et al. 1986).

at $\sim 4200-4300 \AA$. Our Tc search proved to be negative and all sample stars are non-Tc stars (see an example in Fig. A.3). The non-detection of Tc is also suggested by spectrum synthesis around the Tc I $5924 \AA$ line $^{3}$ (see Fig. A.2). Our synthetic spectra suggest that $\mathrm{Tc}$ is not detectable $(\log \varepsilon(\mathrm{Tc})<0.0)$ in our stars, although the Tc I $5924 \AA$ line is not very sensitive to the Tc content.

\section{The super Li-rich massive AGB stars}

The stars SV Cas and R Cen are among the most Li-rich (super Li-rich with $\log \varepsilon(\mathrm{Li})>3-4)$ O-rich (M-type) AGB stars found in our Galaxy ${ }^{4}$. The high Li overabundances observed in these Galactic disc O-rich AGBs are most likely explained by Li-enrichment due to HBB. The evolution of the $\mathrm{Li}$ abundance in HBB-AGB stars strongly depends on several stellar parameters such as progenitor mass, metallicity, mass loss, and convection model (e.g. Mazzitelli et al. 1999; van Raai et al. 2012). The treatment of convection and of mass loss are the most important ingredients in determining the duration of $\mathrm{HBB}$ and the variation of the surface chemistry during the AGB phase. This particularly holds in the range of masses experiencing HBB (Ventura \& D'Antona 2005aa,b). Thus, we compare our observations with up-to-date solar metallicity HBB model predictions with very different prescriptions for mass loss and convection: i) models with the Blöcker (1995) recipe for mass loss and the full spectrum of turbulence convective mixing (HBB-FST; e.g. Mazzitelli et al. 1999); and ii) models with the Vassiliadis \& Wood (1993, VW93) delayed mass loss and the mixing length theory for convection (HBB-MLT; see discussion in Karakas et al. 2012).

The temporal variation of the surface Li abundance in up-todate HBB-FST models of $3.5 M_{\odot} \leq M \leq 6 M_{\odot}$ is displayed in Fig. 1 (left panel). The new models reach higher Li values compared to those previously published by Mazzitellli et al. (1999) in which only the $6 M_{\odot}$ model reached $\log \varepsilon(\mathrm{Li})>4$. The corresponding Li predictions from the most recent HBB-MLT models (Karakas et al. 2012) are very similar with the exception

\footnotetext{
3 The atomic parameters were taken from Palmeri et al. (2005).

4 We mote that the O-rich and Tc-poor (M-type) AGB star R Nor was previously found to be extremely enriched in $\mathrm{Li}$ (with $\log \varepsilon(\mathrm{Li})=4.6$ ) by Uttenthaler et al. (2011). The star R Nor (with $T_{\text {eff }}=3000 \pm 100 \mathrm{~K}$ ) is a spectroscopic twin of R Cen. Our spectrum synthesis confirms that $\mathrm{R}$ Nor is a super Li-rich (with $\log \varepsilon(\mathrm{Li})=4.0$ ) AGB with no (or weak) $\mathrm{Zr}(\log \varepsilon(\mathrm{Zr})=2.6 \pm 0.2)$ enhancement. However, the R Nor spectrum does not cover the Rb I lines at 7800 and $7947 \AA$. We expect R Nor to be non-enriched in $\mathrm{Rb}$, being another example of super Li-rich massive AGBs at the beginning of the TP phase (see text).
}

that the $4 M_{\odot}$ model does not become Li-rich (Karakas 2010). Figure 1 (right panel) shows these predictions for a $5 M_{\odot}$ model. There are two obvious differences between the two sets of models. First, the Li minima in the HBB-FST models are deeper than in the HBB-MLT models, probably because of the different efficiency of the convection model. When the TP is on (or has just extinguished), the HBB-FST models are cooler than the HBBMLT models and this stops Li production for a while, leaving room for Li destruction via proton capture. Second, the evolution of the HBB-FST models (and consequently the duration of the Li-rich phase) is shorter than their HBB-MLT counterparts. The stronger HBB predicted by the FST model (Ventura \& D' Antona 2005a) leads to a fast increase in the stellar luminosity. The use of the Blöcker mass loss recipe in the HBB-FST models further enhances the difference in the evolutionary times of the two sets of models (Ventura \& D'Antona 2005b).

Figure 1 shows that $\log \varepsilon(\mathrm{Li}) \sim 4$ is reached during the first TPs. In the HBB-FST scenario this occurs at the 3rd, 5th, and 11 th $\mathrm{TP}$ for the 6,5 , and $4 M_{\odot}$ models, respectively; in the HBB-MLT framework at the 7th and 14th TP for the 6 and $5 M_{\odot}$ models, respectively. The HBB models also predict $3<{ }^{12} \mathrm{C} /{ }^{13} \mathrm{C}<20$ during the super Li-rich phase, as expected. The HBB-FST models with mass below $4 M_{\odot}$ (Fig. 1, left panel) are also expected to become Li-rich but only in the late AGB phase when the many TP and TDU episodes would favour an enhancement of s-process elements like $\mathrm{Rb}$ (and $\mathrm{Zr}$ ), which is not observed (see Sect. 4). In short, we conclude that our observations of super Li-rich AGBs are consistent with AGB stars of 4-6 $M_{\odot}$ at the beginning of the TP phase. The situation may be more complex for the AGB star RU Cyg with $\log \varepsilon(\mathrm{Li})=2$. This star is probably in an inter-pulse period, on its way to becoming a super Li-rich star, or has just been one. An early AGB stage for these blue HBB-AGB stars is also suggested by other observational properties such as the low changing periods, low infrared excess, and no $\mathrm{OH}$ maser emission (Table A.1) and seems to be corroborated by the specific s-process nucleosynthesis experienced by these stars (see below).

\section{The s-process in massive AGBs at the beginning of the thermally-pulsing phase}

Remarkably, the three bluer AGBs display high Li overabundances accompanied by very weak or no $\mathrm{Rb}$ and $\mathrm{Zr}$ enhancements (Table A.1) while the redder OH/IR AGB RU Ari shows a chemical abundance pattern (high $\mathrm{Rb}$ together with no $\mathrm{Li}$ and weak $\mathrm{Zr}$ ) that is typical of Rb-rich massive Galactic AGBs where the ${ }^{22} \mathrm{Ne}$ neutron source dominates the s-process nucleosynthesis (García-Hernández et al. 2006, 2007). It is to be noted here 
that the $[\mathrm{Rb} / \mathrm{Zr}]$ ratios observed in $\mathrm{Rb}$-rich massive $\mathrm{OH} / \mathrm{IR}$ AGBs such as RU Ari are much larger than predicted by the s-process models (see García-Hernández et al. 2009). The authors van Raai et al. (2012) discuss several possibilities (e.g. a failure of the present model atmospheres which may give to $\mathrm{Rb}$ overestimations, condensation of $\mathrm{Zr}$ to dust, etc.) that could explain this $\mathrm{Rb} / \mathrm{Zr}$ mismatch. However, the situation is very different in the super Li-rich massive AGB stars presented here. These stars are very blue (little circumstellar dust, no $\mathrm{OH}$ maser emission) and are not as extreme as the more evolved and Rb-rich AGBs. Thus, the present model atmospheres and the derived abundances in the super Li-rich massive AGBs are expected to be more reliable (see also Sect. 2).

The short-lived element Tc (usually used as an indicator of an intrinsic AGB star, see below), however, is not detected (i.e. $\log \varepsilon(\mathrm{Tc})<0)$ in all sample stars ${ }^{5}$. The lack of Tc can be explained by the operation of the ${ }^{22} \mathrm{Ne}$ neutron source in massive AGB stars. The s-process nucleosynthesis models that better reproduce the large $\mathrm{Rb}$ overabundances observed in these stars are the delayed superwind models by Karakas et al. (2012), where the production of s-process elements is dominated by the ${ }^{22} \mathrm{Ne}$ neutron source. Their solar metallicity 5 and $6 M_{\odot}$ models show that the super Li-rich phase is reached at the beginning of the TP phase, but it is not until much later (i.e. by the 33rd and 30th $\mathrm{TP}$, respectively) that the s-process abundances (e.g. Rb) start to rise. Interestingly, $\log \varepsilon(\mathrm{Tc})<0$ during all AGB evolution. This is shown in Fig. 1 (right panel) where we display the $\mathrm{Rb}, \mathrm{Zr}$, and Tc predictions for a $5 M_{\odot}$ model with a delayed superwind (Karakas et al. 2012). The activation of the ${ }^{13} \mathrm{C}$ neutron source predicts a much higher and faster Tc production (also $\mathrm{Zr}$ production but at a much slower rate), which is not observed. Models that include a ${ }^{13} \mathrm{C}$-rich region (the ${ }^{13} \mathrm{C}$ pocket) in the He intershell of massive AGB stars (see Karakas et al. 2012; Lugaro et al. 2012) predict a fast increase of the Tc abundance at the beginning of the TP phase or during the super Li-rich phase; e.g. $\log \varepsilon(\mathrm{Tc})>0$ by the 13 th TP in a $5 M_{\odot}$ model (see Fig. A.4). The production of Tc depends on the neutron exposure experienced by the intershell material, specifically, if its value is high enough to allow bypassing the first s-process peak at $\mathrm{Sr}, \mathrm{Y}, \mathrm{Zr}$ to reach Tc. The neutron exposure from the ${ }^{22} \mathrm{Ne}$ source builds up in time, pulse after pulse, which is why the Tc abundance linearly increases with the pulse number. On the other hand, the neutron exposure in the ${ }^{13} \mathrm{C}$ pocket is larger and the same in each ${ }^{13} \mathrm{C}$ pocket, which means that $\mathrm{Tc}$ is already produced in the very first ${ }^{13} \mathrm{C}$ pocket. Thus, we conclude that the lack of Tc in massive AGB stars is the consequence of ${ }^{22} \mathrm{Ne}$ being the dominant neutron source at the s-process site, as predicted by Goriely \& Siess (2004).

The detection of Tc in s-process enriched (MS-, S-, SC-, and C-type) AGB stars has been widely used as an indicator of AGB genuineness ("intrinsic" stars), while its non-detection is indicative of binary systems ("extrinsic" stars; see e.g. Van Eck \& Jorissen 1999; Abia et al. 2002). This is correct if the ${ }^{13} \mathrm{C}$ neutron source dominates the s-process nucleosynthesis, as in low-mass $\left(\sim 1-3 M_{\odot}\right)$ AGB stars. However, we have shown here that the use of Tc as an indicator of AGB genuineness is not valid for more massive AGB stars for which the ${ }^{22} \mathrm{Ne}$ is the dominant neutron source. Notably, many O-rich (M-type) Mira-like stars without Tc, among them our sample star R Cen, were found by Little et al. (1987). We propose that an important fraction of these Tc-poor stars may be super Li-rich massive AGBs at the beginning of the TP phase. Indeed, Little et al. (1987) found that ${ }^{12} \mathrm{C} /{ }^{13} \mathrm{C} \sim 8$ for the three Miras without Tc in their sample, which is very near to the equilibrium value expected for strong HBB AGB stars.

Acknowledgements. This work is partially based on observations made at ESO, 65.L-0317(A). D.A.G.H. acknowledges support for this work provided by the Spanish Ministry of Economy and Competitiveness under grants AYA-2011-27754 and AYA-2011-29060. S.U. acknowledges support from the Austrian Science Fund (FWF) under project P 22911-N16. A.I.K. is grateful for the support of the NCI National Facility at the ANU and the ARC for support through a Future Fellowship (FT110100475). M.L. thanks the ARC for support through a Future Fellowship (FT100100305) and Monash University for support through a Monash Research Fellowship. D.L.L. wishes to thank the Robert A. Welch Foundation of Houston, Texas, for support through grant F-634.

\section{References}

Abia, C., Pavlenko, Y., \& de Laverny, P. 1999, A\&A, 351, 273

Abia, C., Busso, M., Gallino, R., et al. 2001, ApJ, 559, 1117

Abia, C.,Domínguez, I., Gallino, R., et al. 2002, ApJ, 579, 817

Alvarez, R., \& Plez, B. 1998, A\&A, 330, 1109

Benson, P. J., Little-Marenin, I. R., Woods, T. C., et al. 1990, ApJS, 74, 911

Blöcker, T. 1995, A\&A, 297, 727

Cameron, A. G. W., \& Fowler, W. A. 1971, ApJ, 164, 111

Deguchi, S., Sakamoto, T., \& Hasegawa, T. 2012, PASJ, 64, 4

García-Hernández, D. A., García-Lario, Plez, B., et al. 2006, Science, 314, 1751

García-Hernández, D. A., García-Lario, Plez, B., et al. 2007, A\&A, 462, 711

García-Hernández, D. A., Manchado, A., Lambert, D. L., et al. 2009, ApJ, 705, L31

Goriely, S., \& Mowlavi, N. 2000, A\&A, 362, 599

Goriely, S., \& Siess, L. 2004, A\&A, 421, L25

Habing, H. J. 1996, A\&ARv, 7, 97

Hawkins, G., Mattei, J. A., \& Foster, G. 2001, PASP, 113, 501

Herwig, F. 2005, ARA\&A, 43, 435

Karakas, A. I. 2010, MNRAS, 403, 1413

Karakas, A. I., García-Hernández, D. A., \& Lugaro, M. 2012, ApJ, 751, 8

Lambert, D. L., Smith, V. V., Busso, M., et al. 1995, ApJ, 450, 302

Lewis, B. M. 1989, ApJ, 338, 234

Little, S. J., Little-Marenin, I. R., Bauer, W. H. 1987, AJ, 94, 981

Lugaro, M., Karakas, A. I., Stancliffe, R. J., \& Rijs, C. 2012, ApJ, 747, 2

Mathews, G. J., Takahashi, K., Ward, R. A., \& Howard, W. M. 1986, ApJ, 302, 410

Mazzitelli, I., D’Antona, F., \& Ventura, P. 1999, A\&A, 348, 846

Palmeri, P., Fischer, C. F., Wyart, J.-F., \& Godefroid, M. R. 2005, MNRAS, 363, 452

Plez, B. 2012, Astrophysics Source Code Library, record ascl:1205.004

Plez, B., Smith, V. V., \& Lambert, D. L. 1993, ApJ, 418, 812

Sackmann, I. J., \& Boothroyd, A. I. 1992, ApJ, 392, L71

Spencer, J. H., Schwartz, P. R., Winnberg, A., et al. 1981, AJ, 86, 392

Tull, R. G., MacQueen, P. J., Sneden, C., \& Lambert, D. L. 1995, PASP, 107, 251

Uttenthaler, S., \& Lebzelter, T. 2010, A\&A, 510, A62

Uttenthaler, S., Hron, J., Lebzelter, T., et al. 2007, A\&A, 463, 251

Uttenthaler, S., Van Stiphout, K., Voet, K., et al. 2011, A\&A, 531, A88

van Raai, M., Lugaro, M., Karakas, A. I., et al. 2012, A\&A, 540, A44

Van Eck, S., \& Jorissen, A. 1999, A\&A, 345, 127

Vassiliadis, E., \& Wood, P. R. 1993, ApJ, 413, 641

Ventura, P., \& D'Antona, F. 2005a, A\&A, 431, 279

Ventura, P., \& D'Antona, F. 2005b, A\&A, 439, 1075

Wood, P. R., \& Zarro, D. M. 1981, ApJ, 247, 247

Pages 5 to 6 are available in the electronic edition of the journal at http://www. aanda. org

\footnotetext{
5 We note that the detection threshold for Tc in AGB stars is $\log \varepsilon(\mathrm{Tc}) \sim 0$ (see Fig. 15 in Goriely \& Mowlavi 2000).
} 
D. A. García-Hernández et al.: Early massive AGBs

Appendix A: Table A.1 and Figs. A.1-A.4

Table A.1. Sample of early massive AGBs: spectroscopic $T_{\text {eff }}$ and derived $\mathrm{Li}, \mathrm{Rb}$, and $\mathrm{Zr}$ abundances ${ }^{a}$.

\begin{tabular}{|c|c|c|c|c|c|c|c|c|c|}
\hline Star & $\begin{array}{l}\text { Gal. Coor. } \\
(1, b)\end{array}$ & $\begin{array}{c}T_{\text {eff }} \\
\mathrm{K}\end{array}$ & $\begin{array}{c}\log \varepsilon(\mathrm{Li}) \\
\log N(\mathrm{Li})+12\end{array}$ & $\begin{array}{c}\log \varepsilon(\mathrm{Rb}) \\
\log N(\mathrm{Rb})+12\end{array}$ & $\begin{array}{c}\log \varepsilon(\mathrm{Zr}) \\
\log N(\mathrm{Zr})+12\end{array}$ & $\mathrm{Tc}$ & $\begin{array}{r}\text { Period }^{b} \\
\text { (days) }^{2}\end{array}$ & $\begin{array}{c}\text { Masers } \\
\mathrm{SiO} / \mathrm{H}_{2} \mathrm{O} / \mathrm{OH}\end{array}$ & Ref. $^{c}$ \\
\hline RU Cyg & $(97.37,+1.20)$ & 3000 & $2.0 \pm 0.5$ & $<2.6 \pm 0.3$ & $2.6 \pm 0.2$ & No & 442 & $\mathrm{No} / \mathrm{No} / \mathrm{No}$ & $1 / 1 / 1$ \\
\hline SV Cas & $(111.83,-9.04)$ & 3000 & $3.5 \pm 0.5$ & $2.6 \pm 0.3$ & $2.7 \pm 0.2$ & No & 456 & $\mathrm{No} / \ldots / \ldots$ & $2 / \ldots / \ldots$ \\
\hline $\mathrm{R} \mathrm{Cen}^{d}$ & $(313.42,+1.21)$ & 3000 & $4.3 \pm 0.5$ & $2.6 \pm 0.3$ & & No & 251 & No/Yes/No & $1 / 1 / 1$ \\
\hline RU Ari & $(161.47,-41.90)$ & 3000 & No & $4.5 \pm 0.7$ & $<2.6 \pm 0.2$ & No & 357 & Yes/Yes/Yes & $3 / 1 / 1$ \\
\hline
\end{tabular}

Notes. ${ }^{(a)}$ The abundance uncertainties represent the formal errors due to the sensitivity of the derived abundances to slight changes in the model atmosphere parameters $\left(\Delta T_{\text {eff }}= \pm 100 \mathrm{~K}, \Delta[\mathrm{M} / \mathrm{H}]= \pm 0.3, \Delta \xi= \pm 1 \mathrm{~km} \mathrm{~s}^{-1}, \Delta \log g=+0.5, \Delta F W H M=50 \mathrm{~m} \AA\right.$ ) for each star. ${ }^{(b)}$ Present-day pulsation periods derived by us from AAVSO data. ${ }^{(c)}$ References for the $\mathrm{SiO}, \mathrm{H}_{2} \mathrm{O}$, and $\mathrm{OH}$ maser observations. ${ }^{(d)} \mathrm{R}$ Cen was not observed around the $\mathrm{ZrO} 6474 \AA$ bandhead, but its spectrum around the weaker (and less sensitive to the $\mathrm{Zr}$ content) $\mathrm{ZrO}$ bands near $6925 \AA$ is identical to the other stars.

References. (1) Benson et al. (1990); (2) Spencer et al. (1981); (3) Deguchi et al. (2012).
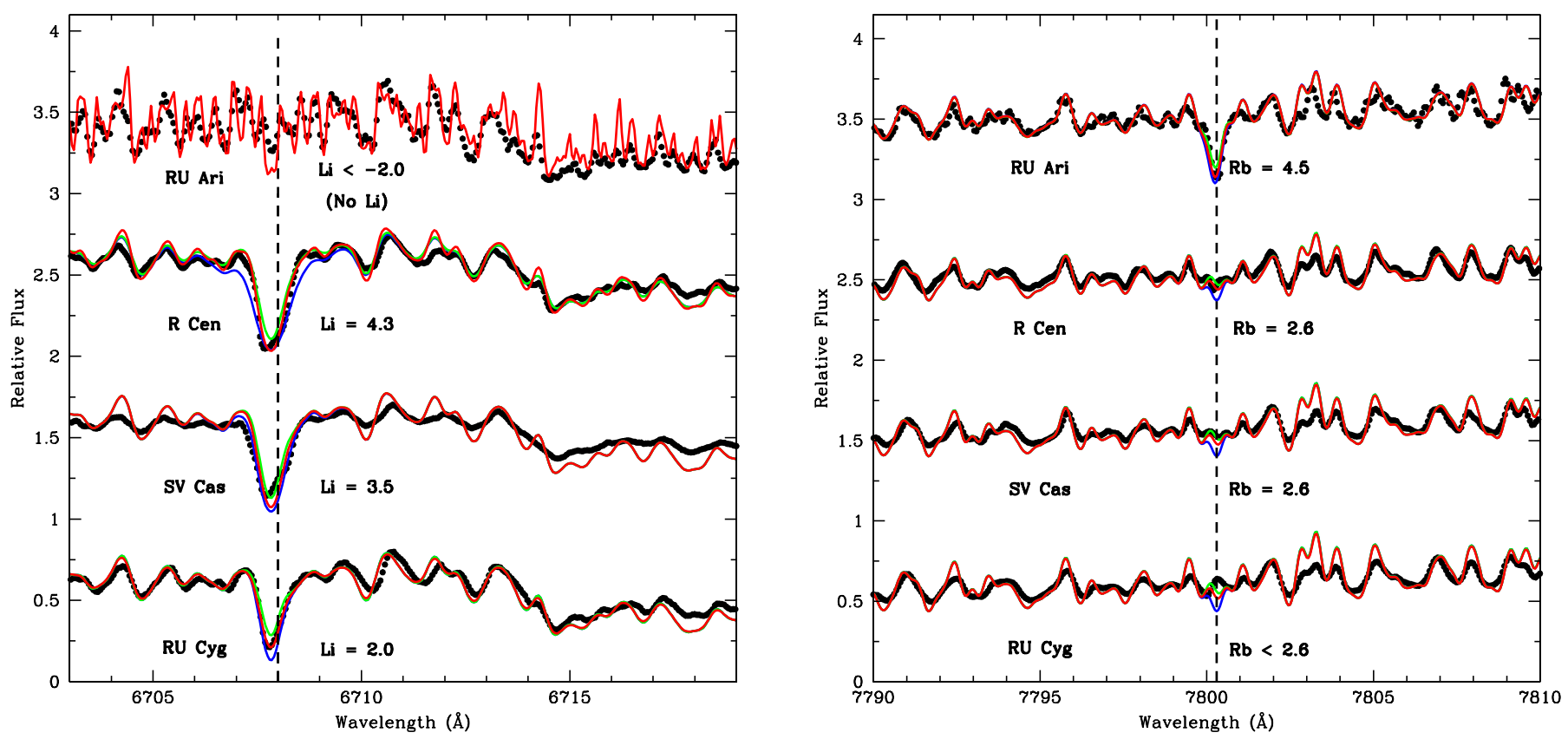

Fig. A.1. High-resolution optical spectra (in black) and best model fits (in red) in the Li I $6708 \AA$ A region (left panel) and Rb I $7800 \AA$ A region (right panel) for the AGB stars RU Ari, R Cen, SV Cas, and RU Cyg. The derived Li and Rb abundances (in the usual scale $\log N(\mathrm{X})+12)$ are indicated. Synthetic spectra obtained for $\mathrm{Li}$ and $\mathrm{Rb}$ abundances shifted $+0.5 \mathrm{dex}$ (in blue) and $-0.5 \mathrm{dex}$ (in green) (these values are \pm 1.0 for $\mathrm{Li}$ in $\mathrm{R} \mathrm{Cen} \mathrm{and}$ $\mathrm{SV}$ Cas) from the adopted values are also shown. We note that $\mathrm{Li}$ is not detected in the extreme OH/IR AGB star RU Ari, which displays a strong $\mathrm{Rb}$ I line that is not detected in the other stars. 

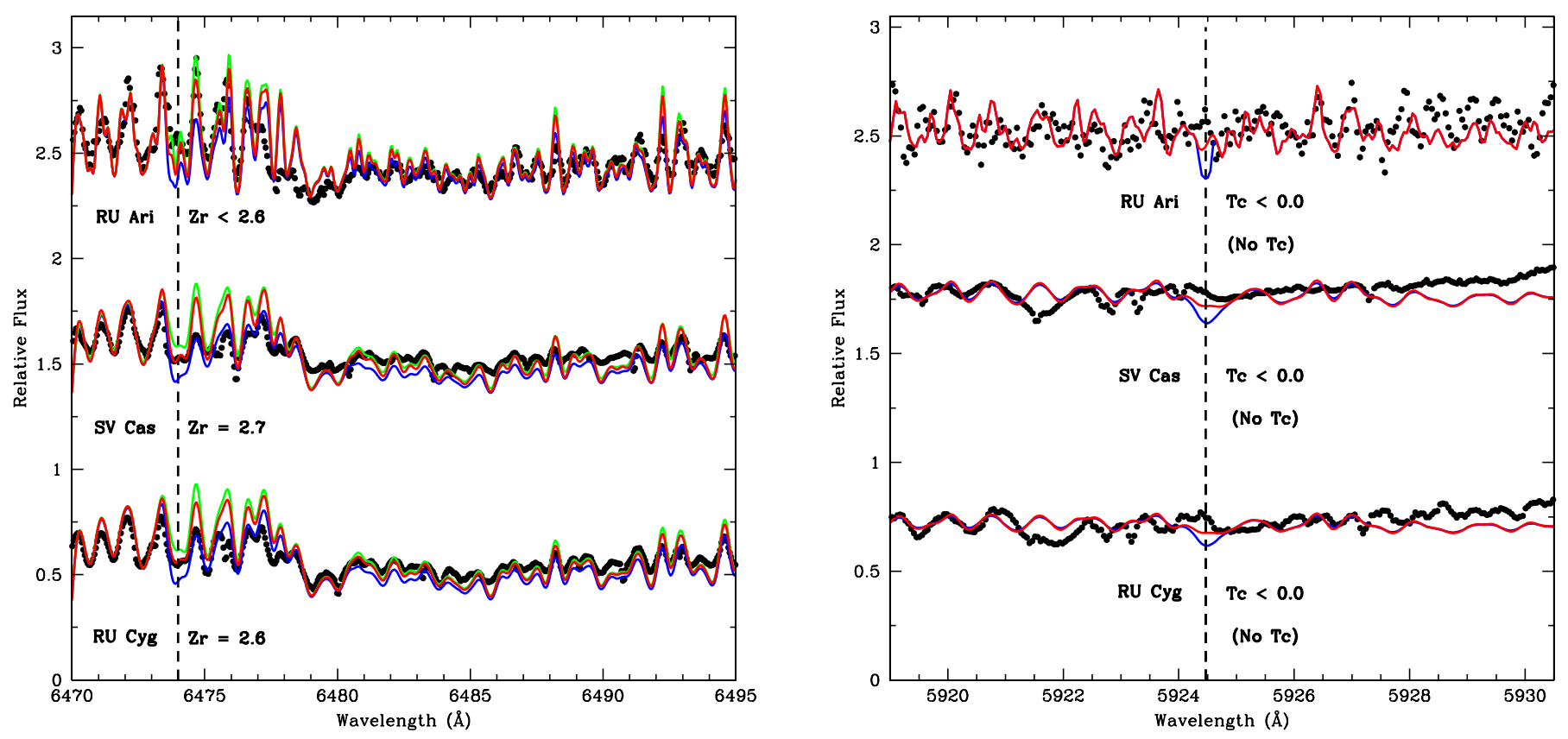

Fig. A.2. High-resolution optical spectra (in black) and best model fits (in red) in the ZrO 6474 A region (left panel) and the Tc I $5924 \AA$ A region (right panel) for the AGB stars RU Ari, SV Cas, and RU Cyg. The derived Zr abundances and Tc upper limits (in the usual scale log $N(\mathrm{X})+12$ ) are indicated. Synthetic spectra obtained for $\mathrm{Zr}$ abundances shifted +0.5 dex (in blue) and -0.5 dex (in green) from the adopted values are also shown. For the less sensitive Tc I $5924 \AA$ line, synthetic spectra obtained for Tc abundances shifted +5.0 dex (in blue) are shown. We note that none of the stars are in our sample is found to be enriched in $\mathrm{Zr}$ or Tc.

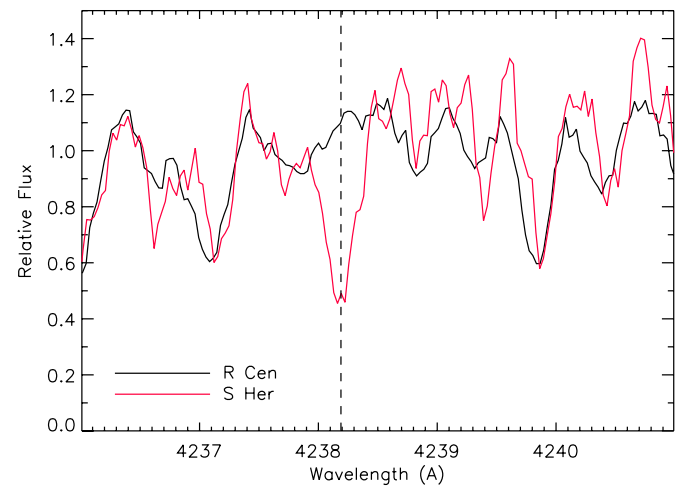

Fig. A.3. High-resolution optical spectra of the non-Tc AGB star R Cen (in black; this paper) and the Tc AGB star S Her (in red; taken from Uttenthaler et al. 2011) around the Tc I line at $4238 \AA$. The wavelength of the Tc line is marked with a dashed vertical line. We note that S Her displays a strong Tc I line that is completely absent in R Cen.

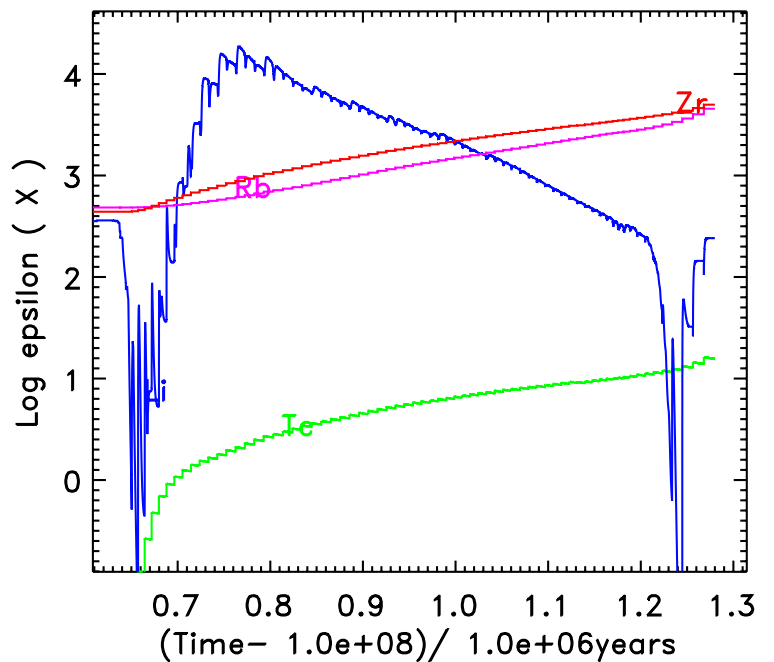

Fig. A.4. Temporal evolution of the $\mathrm{Li}, \mathrm{Rb}, \mathrm{Zr}$, and $\mathrm{Tc}$ abundances as predicted by the HBB-MLT $5 M_{\odot}$ model with a ${ }^{13} \mathrm{C}$-rich region (the ${ }^{13} \mathrm{C}$ pocket) and a delayed superwind (Karakas et al. 2012). We include ${ }^{13} \mathrm{C}$ pocket by inserting protons into the top of the He-intershell (with a mass $=1 \times 10^{-4} M_{\odot}$ ) at the deepest extent of each TDU episode. We refer to Karakas et al. (2012) and Lugaro et al. (2012) for details of this procedure. Tc (also $\mathrm{Zr}$ although at a slower rate) is quickly produced by the ${ }^{13} \mathrm{C}$ neutron source; $\log \varepsilon(\mathrm{Tc})>0$ at the beginning of the super Li-rich phase $(\log \varepsilon(\mathrm{Li}) \sim 4)$. The evolution of $\mathrm{Rb}$ is not greatly affected by the inclusion of the ${ }^{13} \mathrm{C}$ pocket, but $\mathrm{Zr}$ is more affected, being even more abundant than $\mathrm{Rb}$ during the super Li-rich phase. We note that the Tc abundance is an upper limit because the trend of the half-life of ${ }^{99} \mathrm{Tc}$, decreasing with the temperature, is not included. However, the difference would be very small because the ${ }^{99} \mathrm{Tc}$ half-life changes only from terrestrial $0.22 \mathrm{Myr}$ to $0.11 \mathrm{Myr}$ at the temperature of $100 \mathrm{MK}$ typical of the ${ }^{13} \mathrm{C}$ pocket, which means that ${ }^{99} \mathrm{Tc}$ behaves as a stable nucleus during this neutron flux. 